Manuelle Medizin 2009 · 47:173

DOI 10.1007/s00337-009-0692-z

(c) Springer Medizin Verlag 2009

\author{
W. v. Heymann \\ Präsident der FIMM, Bremen
}

\title{
Wissenschaft für die Manuelle Medizin - offen und fair über Grenzen hinweg
}

Wie weiter mit der Wissenschaft nach der Verselbständigung der „Internationalen Akademie für Manuelle und Muskuloskelettale Medizin“? Wird das Ganze zerfallen, wird wieder jeder sein eigenes nationales Süppchen kochen oder mangels Kontakte vertrocknen?

Pfingsten hat in Prag das jährliche Treffen der Akademie stattgefunden. Dies war der erste Testfall für den neuen Wissenschaftsdirektor der Akademie, den Präsidenten der Finnischen Manualmediziner Olavi Airaksinen. Und er hat sich ganz deutlich darum gekümmert, die divergierenden, trennenden Momente $\mathrm{zu}$ bekämpfen. Er hat ganz demonstrativ die DGMM eingeladen, ihr Konzept der Weiterbildung von Ärzten in Manueller Medizin in den Vergleich zu stellen, die Implizierung aktueller Forschungsergebnisse in das Lehrmaterial inhaltlich und pädagogisch zu diskutieren. Das war eine gute Entscheidung, die dem Austausch über die Konzepte sehr geholfen hat und die dazu dienen wird, die Standards in der Weiterbildung weiter zu entwickeln. So klein und überschaubar die Akademie mit ihren knapp 30 wirklichen Aktivisten derzeit erscheinen mag, sie ist heute ein wichtiger Kristallisationspunkt in der wissenschaftlichen Entwicklung der ärztlichen Manualmedizin. Es macht Freude zu sehen, wie sich diese Aktiven kollegial, offen und fair über alle nationalen Grenzen hinweg mit meist noch als Zwischenergebnissen zu beschreibenden Berichten austauschen. Besonders Maxim Baktadze wurde ermutigt, seine SPECT-Untersuchungen über die deutliche Verbesserung der Hirnperfusion nach Manipulation der oberen HWS bei Kopfschmerzpatienten fortzusetzen. Mit diesen Untersuchungen wird uns die inter- nationale Forschung helfen, die äußerst lästigen Anfragen der Kostenträger nach Beweisen für die Wirksamkeit unserer Manuellen Medizin zu beantworten.

\section{Internationales Forum}

Sehr begrüßt wurde deshalb auch der Vorschlag der DGMM, in Fortführung der wissenschaftlichen FIMM- Kongresse nach dem finanziellen Defizit von Bratislava 2004 und der Absage von Zürich 2007 - an einem neuen Design dieses wichtigen internationalen wissenschaftlichen Austausches zu arbeiten. Nächstes Jahr wird wieder der Kongress der DGMM stattfinden, diesmal mit einem begrenzten internationalen Anteil - es wird neben dem deutschsprachigen Hauptteil auch einen englischsprachigen Teil für alle internationalen Aktivisten geben. So sehr sich die internationalen Forscher unter uns Manualmedizinern bemühen, ihre Ergebnisse mit Gleichgesinnten in der begrenzten Akademie zu diskutieren - sie brauchen auch ein größeres Forum, sie brauchen ein Bühne, auf der sie sich einer breiten Kritik aus der Basis der Anwender stellen können. Ohne Zweifel werden besonders die russischen und skandinavischen Freunde eine solche Bühne beim DGMM Kongress nutzen, um ihre aktuellen Ergebnisse vorzustellen. Die mittlerweile insgesamt wieder stabilisierte FIMM sieht sich in der Lage, für ihren Anteil an dem DGMM- Kongress 2010 auch die Verantwortung zu übernehmen.

\section{Europäische Gesellschaft}

Wie soll in diesem Rahmen das Auftauchen einer neuen, Europäischen Gesellschaft für Manuelle Medizin (ESOMM) verstanden werden? Wird es dort wieder nur Aufspaltung von Finanzen und Manpower geben, wieder Konkurrenz und Reibungsverluste? Als Präsident der FIMM denke ich dies nicht. Die Mitglieder der ESOMM sind alle direkt oder mittelbar mit der FIMM verbunden, sie haben nur ein anderes, schnelleres Drehmoment, sie stellen sich den Anforderungen auf wissenschaftlicher und berufspolitischer Ebene direkter. Um den Standard der Prinzipien von Bologna auch für die Ausbildung postgraduierter Ärzte in Manueller Medizin zu erreichen, würde die allen weltweit organisierten Manualmedizinern verpflichtete FIMM zu viel Zeit brauchen - eine nur auf Europa zielende, unbürokratische Aktivistengruppe ist dort schneller und schlagkräftiger. Ich setze daher auf einen positiven SynergieEffekt von FIMM und ESOMM. Auch in diesem Sinne wird der Kongress 2010 eine wichtige Gelegenheit sein, von allen Seiten - Deutschland und international - die Ergebnisse manualmedizinischer Wissenschaft zusammenzutragen. Ich rufe daher schon jetzt dazu auf, die bald erfolgende Einladung zu dem Kongress ganz intensiv mit Beiträgen zum deutschen wie zum internationalen Anteil des Kongresses zu beantworten!

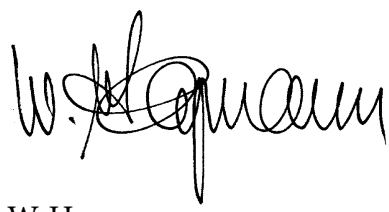

W. Heymann

\section{Korrespondenzadresse \\ Dr. Wolfgang von Heymann \\ Schwachhauser Heerstr. 367 \\ 28211 Bremen \\ heymann@cosit.de}

